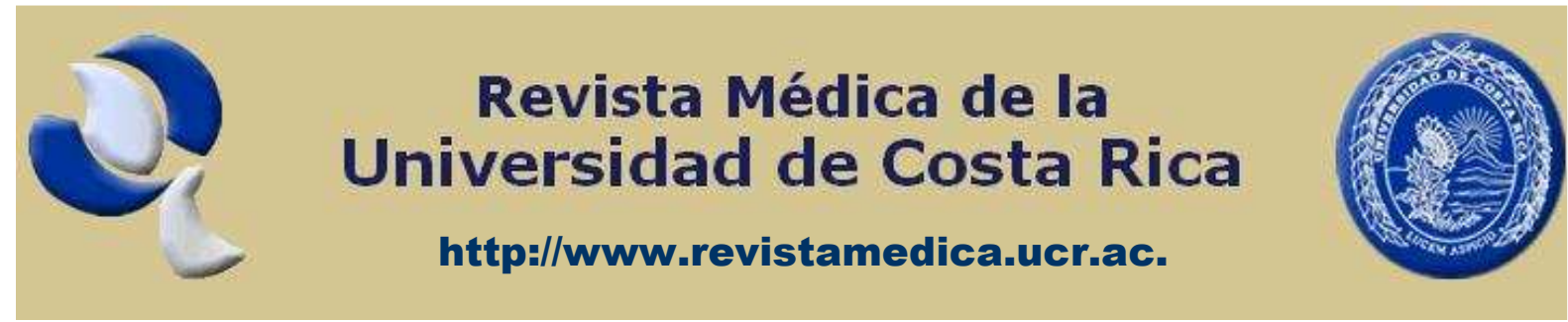

Investigación original

\title{
Manejo fisioterapéutico del dolor lumbar en embarazadas adscritas al Hospital de las Mujeres Dr. Adolfo Carit Eva.
}

\section{Fallas Rodríguez, Diana Elena y Muñoz Acuña, Silvia María.}

Fisioterapeutas, servicios privados.

\section{Resumen}

El presente trabajo tiene la finalidad de realizar una inmersión inicial en la fisioterapia obstétrica, específicamente en el manejo del dolor lumbar en embarazadas. Métodos: Se trabajó con una población total de 32 gestantes en el segundo y tercer trimestre de gestación. Las participantes fueron atendidas por fisioterapeutas, vinculadas temporalmente al servicio de enfermería gineco obstétrica por un periodo de 19 semanas; el cual se extendió de julio a noviembre del 2008, en el Hospital de las Mujeres Dr. Adolfo Carit Eva (HOMACE). A través de éste, se pretende dar respuesta a una necesidad derivada de la condición de gestante, aún no abordada por la salud pública costarricense. Resultado: El $100 \%$ de las variaciones obtenidas para el manejo del dolor lumbar durante el embarazo, se podrían atribuir a las técnicas fisioterapéuticas empleadas. Conclusión: esta intervención proporcionó un manejo fisioterapéutico del dolor lumbar durante el embarazo.

Palabras clave: fisioterapia obstétrica, dolor lumbar, embarazo, técnicas fisioterapéuticas.

Recibido: Marzo 2009. Aprobado: Marzo 2009. Publicado: Marzo 2009.

Revista electrónica publicada por el Departamento de Farmacología de la Escuela de 


\begin{abstract}
The present work pretends to realize an initial inmersion in the obstetric physiotherapy, specifically for handling the lumbar pain in pregnant women. Methods: A total of 32 pregnant women in the second and third trimester of gestation participated. These women were attended by physiotherapists, temporally link to the gineco obstetrics nurse service for 19 weeks; since july to November, 2008, in the Hospital de las Mujeres Dr. Adolfo Carit Eva (HOMACE). Through this, a pregnant women needs pretends to be satisfaced, which the costarrican health system hasn't included in their objetives. Results: The $100 \%$ of the variations for the handling lumbar pain during the pregnancy could be impute to the physiotherapy used techniques. Conclusion: this intervention provided a physiotherapist handling for pregnancy lumbar pain.
\end{abstract}

Keywords: obstetric physiotherapy, lumbar pain, pregnancy, physiotherapy techniques.

Revista electrónica publicada por el Departamento de Farmacología de la Escuela de 


\section{Introducción:}

La fisioterapia obstétrica considera aspectos preventivos y terapéuticos del embarazo, parto y posparto. En países europeos, además de Australia, Sudáfrica y Canadá, existen grandes adelantos en la fisioterapia obstétrica. Los programas para las gestantes incluyen la atención de las manifestaciones físicas, biomecánicas, fisiológicas y emocionales asociadas con el embarazo, entre otros contenidos [1]. La intervención fisioterapéutica en obstetricia es un campo de acción emergente en el continente americano, a excepción de Canadá [2]. En Costa Rica, el sistema de salud pública, posee una normativa dentro de la cual debe atenderse a las embarazadas, "basado en un enfoque de riesgo materno fetal" (detección temprana de algunas alteraciones tales como la anemia, enfermedades de transmisión sexual, diabetes gestacional, hipertensión arterial o inducida por el embarazo y sepsis urinaria) [3]. El abordaje del control prenatal refleja que se considera algunos síntomas como propios del embarazo. Estas condiciones son: dolor lumbar, dolor pélvico, calambres musculares, debilidad de los músculos pélvicos, trastornos de la marcha, trastornos articulares, edema podálico, entre otros [4].

Específicamente para el dolor lumbar durante el embarazo, las causas pueden resumirse en debilidad abdominal, crecimiento uterino, cambios en la postura y biomecánica adoptada y laxitud ligamentaria; las cuales se encuentran íntimamente vinculadas.

En relación con el abdomen, existen dos grupos musculares en los cuales se da la mayor cantidad de cambios. Estos grupos son el anterior, formado por el recto y el piramidal, y el posterior, que abarca el oblicuo mayor, menor y el transverso del abdomen [5].

El grupo anterior es el que más se distiende durante la gestación. Esta distensión puede provocar una diástasis. Cuando se produce este fenómeno, se reduce la capacidad de la mujer de soportar un incremento del peso generado principalmente por el desarrollo del útero. Al afectarse esta capacidad de soporte del útero, su crecimiento produce cambios en la postura [6]. El principal cambio es la hiperlordosis lumbar, la cual a su vez como medio de compensación, provoca hiperlordosis cervical.

La laxitud ligamentaria no se desvincula de las causas anteriores. Las transformaciones que se presentan durante el embarazo tienen influencia hormonal, como por ejemplo la relaxina, una hormona peptídica producida por el cuerpo lúteo, la cual provee a los ligamentos de las articulaciones vertebrales de una mayor laxitud; esto produce aumento de las curvaturas fisiológicas, en especial la lordosis lumbar, acentuando los cambios en la postura [7].

El presente trabajo tiene la finalidad de realizar una inmersión inicial en la fisioterapia obstétrica, específicamente en el manejo del dolor lumbar en embarazadas. A través de éste, se pretende dar

Revista electrónica publicada por el Departamento de Farmacología de la Escuela de 
respuesta a una necesidad derivada de la condición de gestante, aún no

\section{Métodos y materiales:}

El siguiente trabajo es un estudio de tipo observacional transversal exploratorio, diseñado para determinar las características y el comportamiento del dolor lumbar en embarazadas del servicio de enfermería gineco obstétrica del Hospital de las Mujeres Dr. Adolfo Carit Eva (HOMACE). El mismo se prolongó por 19 semanas ubicadas en los meses de julio a noviembre de 2008.

Se analizó una muestra no probabilística con un total de 32 embarazadas. La selección de las personas no dependió de la probabilidad, sino de las condiciones de salud de las embarazadas y del criterio clínico del profesional en enfermería y terapia física.

A cada participante se le dio la posibilidad de recibir cuatro sesiones como máximo, cada una por un lapso de 60 minutos aproximadamente.

Dentro de los criterios de inclusión se citan: embarazadas en el segundo o tercer trimestre de gestación, de 20 a 35 años de edad, con embarazos de bajo riesgo, con referencia extendida por el servicio de enfermería y que posean dolor lumbar. Además, se excluyeron aquellas mujeres que no cumplieran con todos los criterios mencionados, no firmaran el consentimiento informado aprobado por el Comité Local de Bioética, o que presentaran hipertensión arterial y/o diabetes mellitus. abordada por la salud pública costarricense.

La intervención fisioterapéutica se dividió en dos etapas. La primera, de captación, el servicio de enfermería refirió a las usuarias a terapia física, en donde las profesionales a cargo asignaban las fechas de las sesiones. La segunda etapa, de atención, comprende un diagnóstico fisioterapéutico, la aplicación de las técnicas indicadas en embarazo para el manejo del dolor lumbar y una valoración final con el objetivo de establecer las variaciones obtenidas de la aplicación de las técnicas fisioterapéuticas. Durante el diagnóstico se realizó la anamnesis y la exploración física, la cual incluyó la escala de valoración del dolor denominada Escala Gráfica Numérica Visual (EGNV), que consiste en una gráfica que va de los valores cero a 10 , donde cero es no dolor y 10 el peor dolor imaginable. En la aplicación de las técnicas se trabajó con termoterapia superficial, la cual se define como la aplicación del calor con fines terapéuticos [8]; el método terapéutico manual $\mathrm{y} / \mathrm{o}$ instrumental que modifica el estado de los tejidos tratados [9] o más comúnmente conocido como masoterapia; crioterapia que es la aplicación del frío con fines terapéuticos [10]; el contraste (alternando frío y calor) y la estimulación eléctrica transcutánea o en fisioterapia, TENS asimétrico. No se incluyó el calor profundo, ni aquellas corrientes terapéuticas que estuvieran contraindicadas durante el embarazo.

Para llevar a cabo esta intervención se utilizó aceite mineral, toallas de

Revista electrónica publicada por el Departamento de Farmacología de la Escuela de 
papel, paños, sábanas, rollos terapéuticos, compresas calientes y frías, y equipos portátiles de electroterapia.

\section{Resultados:}

Se analizó la información documentada en la anamnesis y exploración física de las embarazadas que cumplieron con todos los criterios de inclusión. Se hizo una comparación cuantitativa, según la escala para la medición de la intensidad del dolor (EGNV), antes y después de la aplicación de las técnicas fisioterapéuticas.

Las características de las 32 gestantes atendidas comprenden la edad (cuadro N. 1) y las semanas de gestación que tenían al momento del diagnóstico (cuadro N. 2).

En cuanto a los antecedentes patológicos personales, se encontró que las embarazadas presentaban gastritis crónica, algún tipo de alergia, colitis, asma y fiebre reumática. Estos antecedentes no se encontraban activos durante la intervención fisioterapéutica.

Al momento de las evaluaciones posturales 19 usuarias mostraron hiperlordosis lumbar, y no se pudo determinar si la causa era una alteración postural del embarazo o si obedecía a una alteración previa a la condición de gestante, ya que para ello habría que contar con antecedentes de una evaluación postural previa al embarazo.

Del total de las sesiones, 21 de 32 mujeres asistieron al tratamiento completo (cuatro sesiones), cuatro gestantes recibieron tres sesiones, otras cuatro se presentaron a dos, y tres recibieron una única sesión. Las participantes que no lograron completar el tratamiento responden a razones de salud, familiares $y$ principalmente laborales.

En el comportamiento del dolor lumbar se describe la semana de aparición del dolor (cuadro N. 3), apreciaciones del dolor según la escala (EGNV) (cuadro N. 4), las características, que podían referir más de una (cuadro N. 5), evolución y frecuencia del dolor, y las acciones para conseguir analgesia.

Al describir la evolución del dolor lumbar inicial, 31 de las 32 embarazadas expresaron que este dolor apareció desde 4 hasta 22 semanas anteriores al diagnóstico, que desde el punto de vista fisioterapéutico se considera una evolución crónica. Una única persona fue atendida con una evolución subaguda, debido a que llegó a la consulta de enfermería con el dolor, por lo se refirió inmediatamente a terapia física.

En relación con la frecuencia de aparición del dolor lumbar inicial, 19 gestantes describieron el dolor lumbar como intermitente. El dolor de tipo permanente lo indicaron 13 gestantes.

Durante el diagnóstico también se indagó sobre las acciones para conseguir analgesia, y 27 participantes describieron lograrla con cambios de posición, ingesta de fármacos, masaje, duchas calientes, reposo y los soportes en el área lumbar. Otras cinco personas refirieron no conseguir analgesia.

Revista electrónica publicada por el Departamento de Farmacología de la Escuela de 
Cuadro N. 1

Gestantes atendidas según rango de edad. HOMACE, julio-octubre, 2008.

\begin{tabular}{lrr}
\hline Edad de la gestante & Frecuencia & Porcentaje \\
\hline $\mathbf{2 0 - 2 4}$ años & 16 & 50,0 \\
$\mathbf{2 5 - 2 9}$ años & 8 & 25,0 \\
$\mathbf{3 0 - 3 4}$ años & 8 & 25,0 \\
Total & 32 & 100,0 \\
\hline
\end{tabular}

Fuente: Elaborado por las autoras, 2008

Cuadro N. 2

Distribución de las embarazadas atendidas según semanas de gestación al inicio de la intervención fisioterapéutica. HOMACE, julio-octubre, 2008.

\begin{tabular}{|l|r|r|}
\hline $\begin{array}{l}\text { Semana } \\
\text { gestación }\end{array}$ & Frecuencia & \multicolumn{1}{l|}{ Porcentaje } \\
\hline $\mathbf{1 6 - 1 9}$ semanas & 8 & 25,0 \\
\hline $\mathbf{2 0 - 2 3}$ semanas & 10 & 31,2 \\
\hline $\mathbf{2 4 - 2 7}$ semanas & 7 & 21,9 \\
\hline $\mathbf{2 8 - 3 1}$ semanas & 5 & 15,6 \\
\hline $\mathbf{3 2 - 3 5}$ semanas & 2 & 6,3 \\
\hline Total & 32 & 100,00 \\
\hline
\end{tabular}

Fuente: Elaborado por las autoras, 2008

Cuadro N. 3

Aparición del dolor lumbar según semana de gestación de las embarazadas atendidas. HOMACE, julio-octubre, 2008.

\begin{tabular}{|l|r|r|}
\hline $\begin{array}{l}\text { Semana aparición dolor } \\
\text { lumbar }\end{array}$ & Frecuencia & Porcentaje \\
\hline Antes embarazo & 6 & 18,8 \\
\hline 4-7 semanas & 2 & 6,3 \\
\hline 8-11 semanas & 4 & 12,5 \\
\hline $\mathbf{1 2 - 1 5}$ semanas & 9 & 28,1 \\
\hline $\mathbf{1 6 - 1 9}$ semanas & 7 & 21,9 \\
\hline $\mathbf{2 0 - 2 3}$ semanas & 1 & 3,1 \\
\hline 24-27 semanas & 3 & 9,4 \\
\hline Total & 32 & 100,0 \\
\hline
\end{tabular}

Fuente: Elaborado por las autoras, 2008

Revista electrónica publicada por el Departamento de Farmacología de la Escuela de 
Cuadro N. 4

Intensidad del dolor lumbar percibido por las embarazadas atendidas durante el diagnóstico. HOMACE, julio-octubre, 2008.

\begin{tabular}{|l|r|r|}
\hline Intensidad del dolor lumbar & Frecuencia & Porcentaje \\
\hline Valor 1 & 1 & 3,1 \\
\hline Valor 3 & 2 & 6,3 \\
\hline Valor 4 & 1 & 3,1 \\
\hline Valor 5 & 8 & 25,0 \\
\hline Valor 6 & 9 & 28,1 \\
\hline Valor 7 & 6 & 18,8 \\
\hline Valor 8 & 4 & 12,5 \\
\hline Valor 10 & 1 & 3,1 \\
\hline Total & 32 & 100,0 \\
\hline
\end{tabular}

Fuente: Elaborado por las autoras, 2008

Cuadro N. 5

Características presentes del dolor lumbar referidas por las embarazadas atendidas. HOMACE, julio-octubre, 2008.

\begin{tabular}{|l|r|r|}
\hline Características presentes & Frecuencia & Porcentaje \\
\hline Punzante & 21 & 65,6 \\
\hline Profundo & 20 & 62,5 \\
\hline Hormigueo & 5 & 15,6 \\
\hline Calambres & 1 & 3,1 \\
\hline Ardoroso & 0 & 0 \\
\hline Quemante & 0 & 0 \\
\hline
\end{tabular}

Fuente: Elaborado por las autoras, 2008

Asimismo, se estableció una comparación de los datos reportados por las embarazadas con tratamiento completo (21), durante la evaluación del dolor lumbar en el momento diagnóstico y de valoración final. Se utilizó una prueba de independencia T-student para datos apareados, para confrontar los estadios inicial y final de las embarazadas atendidas en la intervención fisioterapéutica. cuadro N. 6 muestra esta situación.

Revista electrónica publicada por el Departamento de Farmacología de la Escuela de 
Cuadro N. 6

Comparación del valor inicial de la primera sesión con el final de la cuarta sesión según la intensidad del dolor lumbar en los valores de la

Escala Gráfica Numérica Visual. HOMACE. Julio-Octubre, 2008.

\begin{tabular}{|c|c|c|c|c|}
\hline $\begin{array}{l}\text { Intensidad del dolor } \\
\text { lumbar }\end{array}$ & Media & $\mathbf{N}$ & $\begin{array}{l}\text { Sig. (2- } \\
\text { tailed) }\end{array}$ & $\begin{array}{l}\text { Desviación } \\
\text { estándar }\end{array}$ \\
\hline Primera sesión valor inicial & 5,71 & 21 &, 000 & 2,82 \\
\hline Cuarta sesión valor final & 1,19 & 21 & & \\
\hline Diferencia & 4,52 & & & \\
\hline
\end{tabular}

Fuente: Elaborado por las autoras, 2008.

\section{Discusión:}

En la intervención fisioterapéutica, el dolor lumbar inició en la mayoría de los casos $(78,1 \%)$ durante el cuarto, quinto y sexto mes de gestación (cuadro N. 2), lo cual refleja que en el segundo trimestre del embarazo 25 de las 32 embarazadas atendidas ya presentaban las manifestaciones. Asimismo, sólo un 6,3\% fue atendida entre la semana 32-35. Éste no presentó un número significativo de mujeres debido a que los criterios de inclusión establecían un máximo de 32 semanas de gestación, por lo que se excluyeron a las embarazadas con semanas de gestación superiores.

El cuadro N. 3 muestra que en el grupo de mujeres atendidas, en el $50 \%$ de ellas el dolor apareció entre la semana 12 a 19 de gestación. También debe señalarse que en los casos en que el dolor se presentó antes del embarazo las causas podrían ser el tipo de trabajo que desempeñan las mujeres y la lumbociática. Esta lumbociática se exacerba durante el embarazo por el aumento del peso del útero sobre la región lumbar y sacra [11]. Asimismo, previo a las 12 semanas de gestación las embarazadas refirieron menor presencia de dolor. A partir de esa semana, el dolor se presentó con más regularidad debido a la tensión biomecánica, por aumento del peso del útero en el cual a partir del tercer mes, presenta un crecimiento fetal que aporta 300 gramos en promedio [9]. Esto se suma al aumento de la carga sobre las estructuras de la columna, laxitud ligamentaria, debilitamiento de los músculos abdominales, cambios posturales (lordosis), entre otros [10]. Además, la lordosis pudo estar agravada por un problema preexistente a la condición de embarazo.

Se aprecia en el cuadro N. 4, en cuanto a la intensidad del dolor, que más de un $50 \%$ de las embarazadas lo señalaron con un valor de 5 a 6 en la escala. De la misma forma, es conveniente anotar que en general, las mujeres percibieron ese dolor como propio de su condición de embarazadas. Sin embargo, durante la intervención se les explicó su

Revista electrónica publicada por el Departamento de Farmacología de la Escuela de 
origen, las técnicas recomendadas y su importancia para controlarlo. Cabe destacar que aún cuando las gestantes percibieron el dolor entre 5 ó 6 , estos valores no pertenecen a la sección más alta de la escala (10), pero sí lo consideraron incapacitante, ya que las molestias les impedían llevar a cabo sus actividades de la vida diaria (familiares, sociales, trabajo) de manera regular.

Las características del dolor lumbar más significativas, son tipo punzante y/o profundo (cuadro N. 5). Las características de hormigueo y calambres las indicaron en menor medida y el dolor tipo ardor o quemante, no fue descrito por ninguna usuaria.

Con respecto a la comparación establecida entre los valores iniciales y finales reportados por las embarazadas con tratamiento completo, para la intensidad del dolor lumbar según la EGNV (cuadro N. 6), se obtuvo una media inicial de 5,71 y final de 1,19. Esta diferencia entre los valores refleja que hubo una reducción de la percepción del dolor lumbar en las embarazadas, posterior a la aplicación de las técnicas.

Para esta intervención se estableció un margen de beneficio atribuido al azar del $5 \%$. Sin embargo, para este

\section{Agradecimientos:}

A la Licda. Ingrid Behm, por su aporte científico para la realización de este estudio. caso en particular, este valor corresponde a 0,000 , es decir, el $100 \%$ de los beneficios registrados se debe al manejo fisioterapéutico y no al azar.

A partir de la información obtenida, es pertinente recomendar a las autoridades de salud correspondientes, que el dolor lumbar durante el embarazo no debe ser considerado un síntoma del mismo, sino más bien, una manifestación de los cambios físicos durante el embarazo a la cual debe proporcionársele una solución, como una oportunidad de acceso a una mejor calidad de vida de las mujeres.

\section{Conclusiones:}

Se concluye con los datos recopilados, que la intervención proporcionó un manejo fisioterapéutico del dolor lumbar durante el embarazo. Igualmente, en Costa Rica, al momento de la intervención, las manifestaciones de los cambios físicos del embarazo no son abordados desde la terapia física. Este estudio estableció un primer acercamiento a nivel nacional en el sistema de salud público, en el cual se atendió esta necesidad de las gestantes.

Al Hospital de las Mujeres Dr. Adolfo Carit Eva, por abrir sus puertas para efectuar un estudio pionero en el área de la terapia física. 


\section{Referencias}

1. Stepheson, R y O'Connor, L. Fisioterapia en obstetricia y ginecología". Editorial Mc Graw Hill (2da . Ed.), 2003.

2. Stepheson, $\mathrm{R}$ y O'Connor, L. Fisioterapia en obstetricia y ginecología". Editorial Mc Graw Hill $\left(2^{\text {da }}\right.$. Ed. $), 2003$.

3. Ministerio de Salud. "Normas de Atención Integral de Salud: Atención a la Mujer". 1999.

4. Salas, F y Zelaya, R. "Programa Fisioterapéutico en la Preparación Psicofísica durante el embarazo". Tesis de grado. Escuela de Tecnologías en Salud. Universidad de Costa Rica. 2006.

5. Stepheson, R y O'Connor, L. Fisioterapia en obstetricia y ginecología". Editorial Mc Graw Hill $\left(2^{\mathrm{da}}\right.$. Ed.), 2003.

6. Stepheson, R y O'Connor, L. Fisioterapia en obstetricia y ginecología". Editorial Mc Graw Hill (2 $2^{\text {da }}$. Ed.), 2003.

7. Stepheson, R y O'Connor, L. Fisioterapia en obstetricia y ginecología". Editorial Mc Graw Hill (2da . Ed.), 2003.

8. Martínez, M., Pastor, J y Sendra, F. Manual de medicina física. Editorial Harcourt. (1를 Edición), 2000.

9. Gil, V. Fundamentos de Medicina de Rehabilitación. Editorial Universidad de Costa Rica, (1aㅡ Edición), 2007.
10. Carrie, H. y Lori, B. Ejercicio Terapéutico: Recuperación Funcional. Editorial Paidotribo (1 Edición). 2006.

11. Stepheson, R y O'Connor, L. Fisioterapia en obstetricia y ginecología". Editorial Mc Graw Hill (2 ${ }^{\text {da }}$. Ed.), 2003.

12. Carrie, H. y Lori, B. Ejercicio Terapéutico: Recuperación Funcional. Editorial Paidotribo (1 $\underline{\underline{a}}$ Edición). 2006.

\section{Correspondencia:}

Licda. Diana Elena Fallas Rodríguez, fisioterapeuta, Universidad de Costa Rica. Email: dianitatf@gmail.com, número de teléfono 8837-6586/2460-1505.

Licda. Silvia María Muñoz Acuña, fisioterapeuta, Universidad de Costa Rica. E-mail: silmua18@gmail.com, número de teléfono 88572959/2451-0707.

Revista electrónica publicada por el Departamento de Farmacología de la Escuela de 